\title{
Viewpoint
}

\section{Organisational change and quality of health care: an evolving international agenda}

\author{
Martin McKee, Linda Aiken, Anne Marie Rafferty, Julie Sochalski
}

\begin{abstract}
The nature of organisational change in the health sector

Healthcare reform is now on the agenda of governments in nearly all industrialised countries. ${ }^{1}$ Upward pressure on costs, from such factors as the consequences of new technology and rising public expectations, has collided with downward pressure from economic recession, political unwillingness to increase taxes, and, in the United States, demand for ever greater profits from the corporate providers of health care.
\end{abstract}

The most frequent response to these pressures is to seek ways of limiting costs. These have concentrated largely on hospital care as hospitals continue to consume the largest portion of national health spending ${ }^{2}$ and it has led to a range of major changes in how hospitals are organised.

These organisational changes can be thought of as falling into three broad categories relating to developments outside, within, and between hospitals. The first category considers the interface between the hospital and its external environment, seeking to shift patients currently treated in hospital to other settings. Examples include transfer of diagnostic procedures and minor surgery to ambulatory care, substitution of outpatient care for conditions previously treated on an inpatient basis - such as chronic illness, substance misuse, and depression. The second category considers the hospital's internal environment, seeking ways to increase efficiency by redesigning work, changing staff, reducing inappropriate and thus wasteful care, and by treating patients in less resource intensive ways. Examples include changes in the skill mix of staff, substituting workers with less training for registered nurses, and reductions in ancillary services, intensive care days, and overall duration of stay. The third category relates to the relations between hospitals, asking whether existing configurations of hospital services offer the optimal balance between effectiveness and efficiency on the one hand and accessibility on the other. Examples include mergers of specialist units in neighbouring hospitals, regionalisation of services, and the development of outreach services.

In theory, all of these strategies offer scope to reduce the cost of providing a given package of care while maintaining, or even improving, quality. In practice, however, commentators are increasingly questioning whether this is happening. ${ }^{3}{ }^{4}$ Three main concerns have been voiced. Firstly, can the proposed changes deliver what they set out to? For example, is there empirical evidence that a shift of particular types of patients from hospital to primary care can both reduce costs, and at least maintain quality? Secondly, is organisational change actually being pursued with the aim of reducing costs regardless of quality, with managers concentrating on presentation rather than real changes in quality of care? Thirdly, do the organisational changes being pursued have adverse implications for quality of care by, for example, disrupting existing structures and relations?

Questions about the evidence underpinning these strategies form an enormous topic which cannot be dealt with adequately in the space available. There are, however, several recent reviews - such as those listed on the Cochrane database $\mathrm{e}^{5}$ - considering specific questionssuch as whether the outcome of care is improved by centralising specialist services or whether specific services are best delivered in hospital or in the community. There are four main messages from this research literature. Firstly, it should not be assumed that simply moving a service out of hospital will reduce costs. ${ }^{6}$ Secondly, there are still many gaps in our knowledge of what does or does not work. Thirdly, such changes should be actively managed rather than left to happen by default. ${ }^{7}$ And fourthly, there are many unresolved issues about whether policies found to be beneficial in one setting can be transferred to another. ${ }^{8}$

Concerns about the pursuit of cost savings at the expense of quality also will not be dealt with here, although it is important to note that the magnitude of change in some countries has raised fears about undesirable trade offs being made. This is most obvious in the United States where federal and state legislatures have recently laid down minimum hospital stays after giving birth and limited the ability of hospitals to perform complex procedures such as mastectomies as day cases. ${ }^{9-12}$ These concerns have also led some commentators to view strategies such as total quality management or continuous quality improvement as tools that are primarily designed to "downsize" hospital 
workforces rather than to improve quality. ${ }^{13}$ Such concerns are not confined to the United States, with commentators in Canada ${ }^{14}$ and the United Kingdom also expressing concern about the lack of evaluation of changes.

This paper is consequently confined to the third of these concerns, that the process of organisational change has an adverse effect on quality of care. To consider this issue, we first ask whether major organisational change has actually taken place in leading industrialised countries and, if it has, whether there are any common factors that different national organisational contexts share. Secondly, we examine what is known about how any changes have affected different groups within hospitals. Finally, we explore whether there are reasons to think that such changes might have implications for quality of care.

\section{What has happened in practice?}

Although the need to introduce change is accepted in most countries, the extent to which specific policies have been implemented varies considerably.

The United States, where the healthcare system is dominated by corporate interests in a way that is unique in industrialised countries, has undergone the most change. Between 1980 and 1994, admissions per head of population fell by $26 \%$, with a reduction in duration of stay of nearly one day, to 6.7 days. This led to a $34 \%$ drop in inpatient days per capita. ${ }^{15}$ Although there have been considerable reductions in capacity, both hospital closures and reductions in acute beds have lagged considerably behind the decline in inpatient activity, as shown by the gradual decrease in hospital occupancy, from $76 \%$ in 1980 to just below $60 \%$ in $1995 .{ }^{16}$

Some of the greatest changes have been directed at the organisation of the hospital workforce, reflecting the importance of staff costs, which are typically over half of total hospital operating costs. ${ }^{17}$ Strategies used include reductions in the overall number of full time equivalent employees, and lowering the wage bill by reducing the skill level of the workforce, often with selective targeting of nursing staff. ${ }^{18}{ }^{19}$ Increasingly, hospitals are undertaking major re-engineering programmes in which previous assumptions are discarded and services are rebuilt from scratch. ${ }^{20}$

Canada has also shifted services out of the hospital, with a $25 \%$ decline in admissions per head of population since 1980, although the average duration of stay initially rose from 8.2 to 8.9 days before dropping to 7.8 days in $1993 .^{21}$ Canadian hospital capacity has kept pace with these changes and several provinces have seen considerable declines in hospital beds of up to $20 \%$ during the early 1990 s. The reduction was due in part to the efforts since 1990 to regionalise. ${ }^{22}$ As in the United States and in Western Europe, outpatient services and ambulatory surgery grew substantially.

Since 1990 the United Kingdom has undergone a major programme of health sector reform encompassing a greater emphasis on treatment in primary care, changing methods of working in hospitals, especially in the larger cities, and rationalising facilities. ${ }^{23}$ Unlike the other countries mentioned, admissions to hospitals have risen since 1980, with day surgery cases jumping dramatically since $1991 .^{24}$ Interpretation of these trends is, however, complicated by problems of definition. These changes have been accompanied by a considerable decrease in the average duration of stay. This increasing activity has taken place against a background of a reduction of almost $40 \%$ in available beds between 1980 and 1994 .

Hospital services in The Netherlands also contracted between 1980 and 1995. The admission rate per head of population dropped substantially, and the average duration of stay, although still higher than in North America, decreased by roughly $30 \% .^{2}$ Capacity dropped sharply after hospital consolidation that led to a reduction in both hospitals and beds ${ }^{25}$ similar to that seen in some Canadian provinces. Outpatient and day care services simultaneously rose considerably over this same period.

Germany experienced a growth in bed capacity in the 1960 s and 1970 s and the system of financing in former West Germany during the 1970s and 1980s led to much higher hospital admission rates and substantially longer durations of stay than in comparable countries. ${ }^{2}$ Although admission rates were lower in the former German Democratic Republic, durations of stay were also long. Since the 1990s acute hospital beds per capita have been declining in both parts of Germany, as has duration of stay. ${ }^{26}$ Before 1993 hospitals were prohibited from providing outpatient and ambulatory care services. Subsequently hospitals have slowly moved to develop expanded services. Current financing reforms seek to reduce inpatient activity and shift care to the outpatient sector.

In summary, major organisational changes have taken place in hospitals in many countries. These changes have certain common features - such as a shift from hospital care to other settings, restructuring the internal hospital environment, and rationalising the provision of hospital care. However, there are also differences in both the nature and magnitude of change. For example, reductions in hospital capacity seem to have played more important parts in Canada, The Netherlands, and the United Kingdom than in the United States. In contrast, in the United States the characteristics of the hospital workforce has received more attention than elsewhere.

The common goal of all these countries, to contain the costs of care, has remained elusive. Each country has struggled to have healthcare spending "follow the patient" out of the hospital and into less expensive sites, or to reduce the resource intensity with which patients are treated, thus reducing the rate of growth of total health expenditures without adversely affecting health outcomes. These efforts have had limited success. The proportion of healthcare expenditure accounted for by hospitals has remained high, leading to proposals for even more radical thinking about the delivery of care within hospitals. For example, in the United Kingdom the reduction in beds is likely to 
accelerate considerably in the next few years as a new system of private financing of hospitals is introduced. ${ }^{27}$ This system, which has the advantage to the government of spreading payments over many years, is, in the long term, much more expensive than the existing system, and as a result most of the proposals under it will lead to a local reduction of about $50 \%$ in available beds. As a result, it seems likely that the pace of change in recent years in most countries is likely to continue.

\section{Who has been affected by the process of} organisational change?

The delivery of patient care involves a wide range of staff. In seeking any impact of organisational change on patient care it will be helpful to know whether some groups have been affected more than others. Abbott has argued that, in a period of change, those groups that can classify themselves as professions are able to appeal to societal values and to constituencies outside the organisation to protect their working conditions and thus can deflect pressures for change on to others. ${ }^{28}$ This would suggest that, for example, doctors would be relatively protected.

Evidence of the impact of change derives largely from the United States, partly because its organisational changes were earlier than in other countries, ${ }^{29}$ but also because the United States has had a stronger tradition of organisational research.

This argument was examined in a study by Leicht et al based on annual returns from members of the American Hospitals Association between 1980 and 1988, when American hospitals were going through a period of major restructuring. ${ }^{30}$ With the numbers of each employed group as a measure of ability to withstand change, they concluded that physicians were relatively protected in the face of mergers, restructuring, and instability whereas both administrators and, especially, nurses were adversely affected. Instability and decline were also associated with a greater use of nursing assistants.

More recent evidence has supported the conclusion that nurses have been affected most by the organisational change that has taken place in American hospitals. Aiken et al have reviewed data from the annual surveys of the American Hospital Association and shown that, between 1981 and 1993, despite an overall increase in the hospital workforce of $11.3 \%$, the number of nurses employed has fallen by $7.3 \% .^{31}$ When examined in more detail this shows relative stability in the ratio of professionally qualified nurses to patients, after adjustment for changing case mix, but a considerable reduction in nurses without professional qualifications, giving rise to concerns about the availability of nursing support staff.

This pattern has also been described by Brannon, who noted how American hospitals introduced a system of primary nursing in the 1980 s, replacing the earlier team system in which smaller numbers of professionally qualified nurses would manage others with lower level qualifications. ${ }^{13}$ This is, however, now changing to a system that resembles the former team model, as professionally qualified nurses are being replaced by less qualified staff. $\mathrm{He}$ cites an example of one Californian hospital that sought to reduce its complement of professional nurses by up to $50 \%$, redesignating them as "care coordinators".

The changes seen in the United States are mirrored, to some extent, in other countries. For example, in Canada, the nursing workforce is now much more highly qualified than in the past, although this has been associated with a smaller number of middle managers or specialist roles, and with a failure of wages to keep pace with those in other sectors. ${ }^{32}$ In general, however, there is much less research in countries outside the United States. ${ }^{33}{ }^{34}$

In summary, the available literature on the impact of organisational change in hospitals is largely from the United States. As already noted, the nature of these changes and the context within which they have taken place have been different from those in Europe, with a much greater part being played by profit seeking corporations and more emphasis on restructuring of the hospital workforce than on hospital capacity. None the less, the much greater impact of organisational change on nurses than on physicians is consistent with what was predicted by theory relating to professions and it seems likely that nurses will also be most affected by changes in other countries.

\section{Do changes in the nursing workforce matter?}

To answer this question it is necessary to examine what is known about the relation between organisational factors and outcome. Over the past three decades there have been many publications on correlates of differential hospital mortality, and several organisational and provider related characteristics have been found to be associated with differential mortality. For many of these factors, including teaching status, ownership, size, financial status, and location, the findings about the nature and strength of that association have been inconsistent across studies. ${ }^{35}$ In contrast, nurse staffing variables-such as nurse to patient ratios and nursing skill mix-are consistently found to be significant correlates of inpatient mortality. ${ }^{36-39}$

Although most of this research has focused on staffing, there is a smaller body of research suggesting that the relation between staffing and patient outcomes is influenced by organisational features of hospitals that affect what nurses do.

One such study drew on research that had identified 39 hospitals-so called "magnet" hospitals - that were widely regarded by nurses as offering a good environment in which to practice nursing, ${ }^{35}$ that were characterised by greater nursing autonomy and better relations between doctors and nurses, and were identified in a process that explicitly did not consider outcome. These hospitals were matched with 195 controls sharing the same characteristics. 
After adjustment for severity, the "magnet" hospitals achieved a significant $4.6 \%$ lower inpatient mortality. This difference persisted after taking into account such factors as board certification of physicians and availability of technology.

Other research has strengthened the evidence of a link between professional and organisational satisfaction and outcome, identifying improvements in perceived patient outcome in a culture that was supportive and encouraged flexibility. ${ }^{40}$ Organisational and professional job satisfaction among nurses was used to predict process measures of quality of care $^{41}$ and to differentiate intensive care with apparently good and bad results. This was done on the basis of certain practices - such as the presence of a patient centred culture, strong medical and nursing leadership, effective communication and collaboration, and an open approach to resolution of conflict and problem solving. ${ }^{42}$

This evidence provides an empirical link between professional and organisational satisfaction among nurses and differential hospital mortality. There then arises the question of whether such satisfaction has been affected by organisational change. Some evidence emerges from a study undertaken in Beth Israel Hospital in Boston, Massachusetts between 1993 and 1994. In this period the hospital's operating budget fell by $7.5 \%$ and two wards were closed. Although there were no redundancies, 151 of over 1000 nursing posts were lost. At the same time there were certain other changes, such as a pay freeze, but also the introduction of a programme of integrated clinical practice that was designed to improve communication and career development. ${ }^{43}$ The authors reported a decline in most aspects of satisfaction among nurses over the study period, with low satisfaction most closely associated with perceived heavy workloads and poor communication.

This evidence is supported by research in other hospitals undergoing change, both in the United States ${ }^{44}$ and in Canada, ${ }^{45}$ where new structures and procedures have been shown to give rise to reductions in staff satisfaction, many of which reflect difficulties in communication.

In summary, there is some empirical evidence of an association between organisational change in the United States and nurse satisfaction, and rather more evidence of an association between nurse satisfaction and measures of quality of care.

\section{Where now?}

The changing role of the hospital is one of the major issues facing health systems in all industrialised countries. Each is examining ways of reducing the cost of hospital care. In those countries that have made the greatest changes there are now major concerns that cost containment is being pursued at the expense of quality, with commentators increasingly questioning the limits to which intensification of economies can be pursued.

The research base against which policy makers must make decisions about the appropriate balance of hospitals and primary care, or about the nature of the hospital workforce, is extremely weak, although what evidence does exist indicates that any adverse effects are most likely to be seen by looking at the contribution to patient care made by nurses. Furthermore, the limited evidence that does exist suggests that fears about reduced professional and organisational satisfaction and their impact on quality of care, at least in the United States, may be justified.

What is now needed is a concerted international research effort that will seek to answer these questions. ${ }^{3}$ In its absence there is a real danger that decisions will be made on the basis of what is measurable-such as costs-rather than quality of care. This could lead to hospitals becoming pressure chambers rather than safe spaces for clinical care.

This work draws on discussions held at a meeting held in Bellagio,Italy, in November 1996, and was supported by the Rockefeller Foundation, and on research undertaken in the United Kingdom as part of a project funded by the Nuffield Provincial Hospitals Trust.

1 World Health Organisation. European health care reform: analysis of current strategies. Copenhagen: WHO, 1997.

2 US Congress. Office of Technology Assessment. Hospital financing in seven countries (OTA-BP-H-148). Washington, DC: US Government Printing Office, 1995.

3 Sochalski J, Aiken LH, Fagin CM. Hospital restructuring in the United States, Canada, and Western Europe: an outcomes research agenda. Med Care 1997;35:13-25.

4 Pollitt C. Business approaches to quality improvement: why they are hard for the NHS to swallow. Quality in Health Care 1996;5:104-10.

5 The Cochrane Library. The Cochrane Collaboration; issue 4. Oxford: Update Software; 1997.

6 Hensher M, Fulop N, Hood S, et al. Does hospital at home make economic sense? Results of an economic evaluation of early discharge hospital at home for othopaedic patients in three areas of West London. $\mathcal{F} R$ Soc Med 1996;89:54851

7 Waghorn A, McKee M, Thompson J. Surgical outpatients: challenges and responses. Br F Surgery 1997;84:300-7.

8 Rose R. Lesson drawing in public policy: a guide to learning across time and space. Chatham, NJ: Chatham House, 1993.

9 Baer ED, Fagin CM, Gordon S, eds. Abandonment of the patient. New York: Springer, 1996.

10 Schindul-Rothschild J, Berry D, Long-Middleton E. Where have all the nurses gone? Final results of the AJN patient care survey. American fournal of Nursing 1996;96:24-8.

11 Gordon S. What nurses stand for. The Atlantic Monthly 1997;279:81.

12 Institute of Medicine [editorial]. Nursing staff in hospitals and nursing homes: is it adequate? Washington, DC: National Academy Press, 1996.

13 Brannon RL. Restructuring hospital nursing: reversing the trend toward a professional workforce. Int $\mathcal{F}$ Health Serv 1996;26:643-54

14 Hall LM. Staff mix models: complementary or substitution roles for nurses. Nursing Administrative Quarterly 1997;21: $31-9$

15 American Hospital Association. Hospital stat: emerging trends in hospitals (1995-96 edition). Chicago IL: AHA, 1995.

16 Prospective Payment Assessment Commission. Are and the American health care system: report to Congress, fune 1996. Washington, DC: PROPAC, 1996.

17 Greene J. Does integration really cut costs? Mod Health Care 1997;27:34

18 McArthur JH, Moore FD. The two cultures and the health care revolution: commerce and professionalism in medical care revolution: commerce

19 McCloskey JC, Mass M, Huber DG, et al. Nursing management innovations: a need for systematic evaluation. Nursing Economics 1994:12:35.

20 Tunick PA, Etkin S, Horrocks A, et al. Reengineering a cardiovascular surgery service. Foint Commission fournal on Quality Improvement 1997;23:203-16.

21 Canadian Institute for Health Information. Selected data for acute hospital care. Ottowa: CIHI, 1996.

22 Reamy J. Health service regionalization in New Brunswick, Canada: a bold move. Int $\mathcal{F}$ Health Serv 1995;25:271

23 Maynard A, Bloor K. Introducing a market to the United Kingdom's National Health Service. $N$ Engl $7 \mathrm{Med}$ 1996;334:604-8.

24 Harrison AJ. Hospitals in England: impact of the 1990 National Health Service reforms. Med Care 1997;35:5061 .

25 Maarse H, Mur-Veeman I, Spreeuwenberg C. The reform of hospital care in The Netherlands. Med Care 1997;35:26 39. 
26 Busse R, Schwartz FW. Financing reforms in the German hospital sector: from full cost cover to prospective case fees. Med Care 1997; $35: 40-9$.

27 Pollock AM, Dunnigan M, Gaffney D, et al. What happens when the private sector plans hospital services for the NHS: three case studies under the private finance initiative. BMF 1997;314:1266-71.

28 Abbott A. The system of professions: an essay on the division of expert labour. Chicago, IL: University of Chicago, 1988

29 Alexander J. D'Aunno T. Transformations of institutional environments: perspectives on the corporatization of US health care. In: Mick S, ed. Innovations in health care delivery: new insights into organizational theory. San Francisco, CA: Jossey-Bass, 1990

30 Leicht KT, Fennell ML, Witkowski KM. The effects of hospital characteristics and radical organizational change on the relative standing of health care professions. F Health Soc Behav 1995;36:151-67.

31 Aiken LH, Sochalski J, Anderson GF. Downsizing the hospital nursing workforce. Health Aff (Millwood) 1996;15:88pital

32 Baumgart AJ. Hospital reform and nursing labor market trends in Canada. Med Care 1997;35:124-31

33 Buchan J, Hancock C, Rafferty AM. Health sector reform and trends in the United Kingdom hospital workforce. Med Care 1997;35:143-50.

34 Muller-Mundt G. Trends in hospital restructuring and the impact on the workforce in Germany. Med Care 1997;35 132-42.

35 Aiken LH, Smith H L, Lake ET. Lower medicare mortality among a set of hospitals known for good nursing care. Med Care 1994;32:771-87.

36 Hartz AJ, Krakauer H, Kuhn EM, et al. Hospital characteristics and mortality rates. $N$ Engl f Med 1989;321:1720-5.
37 Shortell SM, Hughes EF. The effects of regulation, competition, and ownership on mortality rates among hospital inpatients. N Engl F Med 1988;318:1100-7.

38 Georgopoulos BS, Mann FC. The community general hospital. New York: Macmillan, 1962.

39 Al-Haider AS, Wan TTH. Modeling organizational determinants of hospital mortality. Health Serv Res 1991;26:303.

40 Shortell SM, O'Brien JL, Carman JM, et al. Assessing the impact of continuous quality improvement/ total quality management: concept versus implementation. Health Serv Res 1995;30:377-401.

41 Leveck ML, Jones CB. The nursing practice environment, staff retention, and quality of care. Res Nurs Health 1996;19:331-43.

42 Zimmerman JE, Shortell SM, Rousseau DM, et al. Improving intensive care: observations based on organisational case studies in nine intensive care units: a prospective, multicenter study. Crit Care Med 1993;21:1443-51.

43 Davidson $\mathrm{H}$, Folcarelli $\mathrm{PH}$, Crawford S, et al. The effects of health care reforms on job satisfaction and voluntary turnover among hospital-based nurses. Med Care 1997;35: 634-45.

44 Rubenstein L V, Lammers J, Yano EM, et al. Evaluation of the VA's pilot program in institutional reorganization toward primary and ambulatory care: part II, a study of organizational stresses and dynamics. Acad Med 1996;71: 784-92.

45 Armstrong-Stassen M, Cameron SJ, Horsburgh ME. The impact of hospital downsizing on the job satisfaction of nurses. Canadian fournal of Nursing Administration 1996;9: $8-32$. 